\title{
Serum Levels of S100A11 and MMP-9 in Patients with Epithelial Ovarian Cancer and Their Clinical Significance
}

\author{
Wenjing Li, ${ }^{1}$ Zhumei Cui, ${ }^{2}$ Yan Kong, ${ }^{1}$ Xiangyu Liu, ${ }^{1}$ and Xiangyu Wang $\mathbb{D}^{1}$ \\ ${ }^{1}$ Department of Gynecology, The Affiliated Hospital of Qingdao University, Qingdao University, Qingdao, China \\ ${ }^{2}$ Department of Gynecology, The Affiliated Hospital of Qingdao University, Qingdao, Shandong Province, China \\ Correspondence should be addressed to Xiangyu Wang; 15863061517@163.com
}

Received 22 August 2020; Revised 29 December 2020; Accepted 7 January 2021; Published 4 March 2021

Academic Editor: Luca Giannella

Copyright (C) 2021 Wenjing Li et al. This is an open access article distributed under the Creative Commons Attribution License, which permits unrestricted use, distribution, and reproduction in any medium, provided the original work is properly cited.

\begin{abstract}
Objective. To investigate the serum levels of calgizzarin (S100A11) and matrix metalloproteinase-9 (MMP9) in patients with epithelial ovarian cancer (EOC) and determine their clinical significance. Methods. Serum levels of S100A11 and MMP9 were detected in patients with EOC, patients with benign ovarian tumor, and healthy women. The correlation between the two markers and clinicopathological characteristics of ovarian cancer was analysed. Results. The serum levels of S100A11 and MMP9 in patients with EOC were higher than those in patients with benign ovarian tumor and in healthy women, and the expression levels of S100A11 and MMP-9 were positively correlated. S100A11 and MMP-9 were correlated with tumor staging, postoperative residual foci, ascites volume, serum CA125 level, chemotherapy response, and lymph node metastasis, while S100A11 and MMP-9 were not associated with the bilevel classification, histological type, age, and degree of differentiation. Conclusion. S100A11 and MMP-9 were both highly expressed in the serum of patients with EOC and were associated with cancer development, invasion, and metastasis. Therefore, they can be used as an important reference maker in the diagnosis and treatment of ovarian cancer.
\end{abstract}

\section{Introduction}

EOC is the most common type of ovarian cancer; it has a high degree of malignancy and an insidious onset, and the long-term survival rate remains at 20-30\% [1]. Most patients are diagnosed at the late stage of cancer and miss the optimal timing for surgery. Therefore, the search for definitive early diagnostic indicators is of great significance for the diagnosis, treatment, and prognosis of patients with ovarian cancer. As in the case of other malignant tumors, tumor invasion and metastasis are important factors affecting the prognosis of patients with EOC. The occurrence and development of EOC is a multifactorial, multistage, complex process that is affected by many factors [2]. The origin and pathogenesis of ovarian cancer have not yet been elucidated. Further understanding of the molecular mechanisms of ovarian cancer may lead to the more effective development of treatment strategies.

S100A11 is a multigene family of EF-hand calciumbinding proteins. S100A11 is an important member of the
S100 protein family. It is mainly involved in the regulation of cell-signaling pathways but also plays a role in cell growth, proliferation, differentiation, and apoptosis and is closely associated with tumors. It has been reported that high expression of the S100A11 gene in some tumors can upregulate the expression of MMP-9, thus promoting tumor invasion [3]. MMP-9 is a member of the matrix metalloproteinase family. As an important proteolytic enzyme, MMP-9 is involved in tumor invasion and metastasis. Through the degradation of the extracellular matrix, it accelerates the infiltration of cancerous cells into surrounding tissues [4]. Currently, there are few studies on the expression of S100A11 and MMP9 in EOC, and the clinical correlation between S100A11 and MMP9 is also not clear [5]. In this study, enzyme-linked immunosorbent assay (ELISA) was used to detect the levels of serum S100A11 and MMP9 in patients with EOC, patients with benign ovarian tumor, and healthy women to explore the correlation between the two potential markers and their relationship with EOC invasion and metastasis. 
TABLE 1: Expression levels of S100A11 and MMP-9 in the serum of each group.

\begin{tabular}{lccr}
\hline Group & No. of patients & S100A11 (ng/mL) & MMP-9 (ng/mL) \\
\hline Healthy control group & 25 & $5.02(1.54-7.19)$ & $89.63(7.28-209.47)$ \\
Benign group & 30 & $8.41(5.12-14.85)$ & $122.95(54.99-256.26)$ \\
Malignant group & 111 & $14.54(4.46-38.86)$ & $189.71(35.21-688.99)$ \\
\hline
\end{tabular}

The Kruskal-Wallis test was used to compare different groups: the serum levels of S100A11 in malignant group compared with control group and benign group, $P<0.05$; the serum levels of MMP-9 in malignant group compared with control group and benign group, $P<0.05$.

\section{Materials and Methods}

2.1. Patient Samples. A total of 111 patients with EOC who were diagnosed and treated at the Department of Obstetrics and Gynaecology at the Affiliated Hospital of Qingdao University, China, between November 2010 and January 2020 were included in this study. Thirty patients with benign ovarian tumors and 25 healthy women who underwent physical examinations during the same period were included as controls. The subjects were between 34 and 74 years old, with a median age of 51 years. The pathological diagnosis of all EOC patients was confirmed by two experienced pathologists. Surgical staging was carried out according to the staging criteria of the International Federation of Gynaecology and Obstetrics (FIGO) [6]. None of the subjects had a history of chemotherapy, radiotherapy, or immunotherapy before specimen collection. The samples were classified based on clinicopathological parameters, including age at initial diagnosis, FIGO stage, histological subtype, histological grade, ascites volume, serum CA125 level, postoperative residual tumor mass size, and chemotherapy response. Aside from one stage I G1 patient, the EOC patients received cytoreductive surgery and paclitaxel plus platinum-based chemotherapy. Patients were grouped according to the assessment of their chemotherapy response [7] and the dualistic theory of ovarian cancer [8]. This study was approved by the Ethics Committee of the Affiliated Hospital of Qingdao University (approval number: QYFYWZLL 26079). All patients or their relatives signed written informed consent.

2.2. Method. Early in the morning before surgery, $8 \mathrm{~mL}$ of fasting peripheral blood was collected from the subjects and centrifuged at $2000 \mathrm{~g}$ for $10 \mathrm{~min}$. The separated serum was stored in a $-80^{\circ} \mathrm{C}$ freezer for subsequent measurement. The levels of S100A11 and MMP9 in peripheral blood were measured using the MMP-9 ELISA kit from R\&D (USA) and the S100A11 ELISA kit from BioVendor.

2.3. Statistical Analysis. SPSS 25.0 was used for data analysis. The data obtained in this study were measurement data. The Shapiro-Wilk test was used to assess the distribution of normality. Since the data were not normally distributed, median (P25-P75) was used for the data description. The KruskalWallis or Mann-Whitney $U$ test was used to compare and analyse different groups. The Bonferroni correction method was used for comparisons between groups. Spearman's rank correlation analysis was used for correlation analysis. $P<0.05$ was considered statistically significant.

\section{Results}

3.1. Expression Levels of S100A11 and MMP-9 in the Serum of Each Group. The serum levels of S100A11 and MMP-9 in the malignant group were significantly higher than those in the benign group and the healthy control group $(P<0.05)$. The levels of S100A11 and MMP-9 in the peripheral blood of the benign group were significantly higher than the levels in the healthy control group $(P<0.05)$ (Table 1$)$.

3.2. Correlation between S100A11, MMP-9, and Other Clinicopathological Features of EOC Patients. High serum S100A11 levels in EOC patients were closely related to tumor pathological stage, postoperative residual foci, ascites volume, serum CA125 level, platinum-based chemotherapy response, and lymph node metastasis $(P<0.05)$. Similarly, high serum MMP-9 levels were also closely related to the aforementioned six factors $(P<0.05)$. Conversely, S100A11 and MMP-9 expression in the peripheral blood of EOC patients was not associated with the bilevel classification or the classification of EOCs, histological type, age, or degree of differentiation $(P>0.05)$. The serum levels of S100A11 and MMP-9 in patients with late-stage (stage III-IV) EOC were higher than those of patients in the early stage (stage I-II); the expression levels of S100A11 and MMP-9 in the serum of patients with lymph node metastasis were higher than the serum levels of patients without metastasis; the expression level of S100A11 and MMP-9 in the serum was high in patients with a large volume of ascites or large residual tumor foci. The expression levels of S100A11 and MMP-9 were higher in chemotherapy-insensitive patients than in chemotherapysensitive patients, and all differences were statistically significant $(P<0.05)$ (Table 2$)$.

3.3. Correlation between Serum S100A11 and MMP-9 in EOC Patients. Spearman's rank correlation analysis showed a significant positive correlation between serum S100A11 and MMP9 in EOC patients (rs $=0.405, P<0.05$ ).

\section{Discussion}

Due to the lack of specific clinical manifestations and effective screening methods, ovarian cancer is difficult to detect and diagnose at an early stage. Most patients have an excellent response to platinum-based drugs at the initial treatment, but almost all patients with advanced ovarian cancer will relapse and will progress to platinum resistance after multiple relapses [9]. Targeted drugs have been written into ovarian cancer treatment guidelines [10] and are expected 
TABLE 2: The relationship between the serum levels of S100A11 and MMP-9 and clinicopathological characteristics in patients with EOC.

\begin{tabular}{|c|c|c|c|c|c|}
\hline Clinical variable & No. of patients & $\begin{array}{l}\text { S100A11 (ng/mL) } \\
\text { Median (range) }\end{array}$ & $P$ value $^{\mathrm{a}}$ & $\begin{array}{l}\text { MMP-9(ng/mL) } \\
\text { Median (range) }\end{array}$ & $P$ value $^{\mathrm{a}}$ \\
\hline All cases & 111 & & & & \\
\hline Age at diagnosis(years) & & & 0.201 & & 0.136 \\
\hline$<51$ & 55 & $11.01(4.46-32.74)$ & & $165.53(35.21-688.99)$ & \\
\hline$\geq 51$ & 56 & $15.60(5.60-38.86)$ & & $199.61(59.01-475.02)$ & \\
\hline Histological type & & & 0.32 & & 0.678 \\
\hline Serous & 80 & $13.41(4.46-32.22)$ & & $180.51(35.21-475.02)$ & \\
\hline Mucinous & 10 & $12.58(7.60-32.75)$ & & $195.80(63.42-688.99)$ & \\
\hline Endometrioid & 13 & $10.75(6.38-38.86)$ & & $151.90(47.22-422.59)$ & \\
\hline Clear cell tumor & 8 & $12.32(8.13-29.86)$ & & $153.26(55.59-248.06)$ & \\
\hline Tumor grade & & & 0.93 & & 0.978 \\
\hline G1 & 8 & $13.02(8.03-22.62)$ & & $182.96(63.42-441.95)$ & \\
\hline $\mathrm{G} 2$ & 23 & $11.71(6.38-32.22)$ & & $156.85(47.22-422.59)$ & \\
\hline G3 & 80 & $13.02(4.46-38.86)$ & & $181.38(35.21-688.99)$ & \\
\hline Lymph node metastasis & & & 0.01 & & 0.007 \\
\hline Negative & 60 & $12.58(4.46-32.75)$ & & $154.03(35.21-688.99)$ & \\
\hline Positive & 51 & $14.24(5.60-38.86)$ & & $204.23(63.47-475.02)$ & \\
\hline FIGO stage & & & $<0.001$ & & $<0.001$ \\
\hline I, II & 30 & $10.00(6.38-29.86)$ & & $139.94(43.82-246.16)$ & \\
\hline III, IV & 81 & $14.15(4.46-38.86)$ & & $199.65(35.21-688.99)$ & \\
\hline Serum CA125 level (U/mL) & & & 0.04 & & 0.002 \\
\hline$<35$ & 26 & $12.32(4.46-31.26)$ & & $154.44(35.21-441.95)$ & \\
\hline$\geq 35$ & 85 & $14.29(6.20-38.86)$ & & $199.59(43.82-688.99)$ & \\
\hline Chemotherapy resistance ${ }^{\mathrm{b}}$ & & & 0.01 & & $<0.001$ \\
\hline Present & 31 & $12.32(4.46-31.26)$ & & $154.44(35.21-441.95)$ & \\
\hline Absent & 79 & $14.29(6.20-38.86)$ & & $199.59(43.82-688.99)$ & \\
\hline Ascites $(\mathrm{mL})$ & & & 0.01 & & 0.045 \\
\hline$<500$ & 51 & $12.32(4.46-31.26)$ & & $154.44(35.21-441.95)$ & \\
\hline$\geq 500$ & 60 & $14.29(6.20-38.86)$ & & $199.59(43.82-688.99)$ & \\
\hline Two-tier system & & & 0.52 & & 0.29 \\
\hline I type & 45 & $12.76(6.38-38.86)$ & & $156.85(47.22-441.95)$ & \\
\hline II type & 66 & $13.19(4.46-32.75)$ & & $183.62(35.21-688.99)$ & \\
\hline Residual tumor size $(\mathrm{cm})$ & & & 0.03 & & 0.002 \\
\hline$<2$ & 86 & $12.76(4.46-38.86)$ & & $167.70(35.21-688.99)$ & \\
\hline$\geq 2$ & 25 & $15.81(5.60-31.17)$ & & $244.50(83.18-344.22)$ & \\
\hline
\end{tabular}

The Mann-Whitney $U$ and Kruskal-Wallis nonparametric tests were used to compare different groups. ${ }^{\mathrm{b}}$ One patient with stage I and Grade I did not undergo chemotherapy.

to make ovarian cancer a controllable chronic disease, though it still cannot be cured. The mortality rate of ovarian cancer remains the highest among gynaecologic tumors. A common challenge facing various treatment regimens for ovarian cancer is the lack of effective biomarkers.

S100A11, also known as S100C or calgizzarin, is an important member of the S100 family [11]. A number of cancers have been reported to involve overexpression of S100A11, including thyroid cancer [12], colon cancer [13], pancreatic cancer [14], and breast cancer [15]. The study of Shankar et al. [16] showed that the reduction or deletion of S100A11 expression could lead to an interaction between cells and E-cadherin protein expression, thereby reducing tumor migration and invasion. S100A11 also has high expression in ovarian cancer cells. Knocking out the S100A11 gene could inhibit the growth, invasion, and migration of ovarian cancer cells [17].

The degradation of the extracellular matrix is a crucial step in the invasion and metastasis of malignant tumors. MMP-9 has the largest molecular weight among the MMPs, and it can degrade almost all the extracellular matrix components, causing weakening of the adhesion between tumor cells and damaging barriers that prevent cancer cell invasion and metastasis, thus providing sufficient space for cancer cells to spread and metastasize to distant regions and promoting tumor growth, infiltration, and metastasis [18]. 
Wood et al. [19] confirmed that MMP-9 is involved in the tumorigenesis, invasion, and metastasis processes of a variety of tumors. Therefore, inhibition of S100A11 and MMP-9 expression may further inhibit tumor invasion and metastasis, providing a promising direction in the diagnosis and treatment of ovarian cancer patients.

The experimental results showed that high serum S100A11 and MMP-9 expression was associated with high CA125 and a large volume of ascites. CA125 is an important biomarker of EOC [20], and ascites is also considered an important indicator in the diagnosis, treatment, and followup of EOC [21]. These results preliminarily confirmed that S100A11 and MMP-9 were associated with CA125 and ascites, suggesting that S100A11 and MMP-9 were closely related to the progression of EOC. The monitoring of S100A11 and MMP-9 levels is helpful for EOC diagnosis, efficacy evaluation, and prognosis evaluation.

Chemoresistance is considered to be the main reason for treatment failure in EOC patients and is an important indicator for determining the prognosis of EOC in clinical practice [22]. Studies have shown that in ovarian cancer cells with high MMP-9 expression, the combined use of MMP9/MMP2 inhibitors and cisplatin could significantly enhance cytotoxicity, confirming that MMP9 is a potential therapeutic target for drug-resistant ovarian cancer [23]. S100A11 has also been confirmed to be associated with drug resistance in tumors. Zagresjzzaya et al. [24] found that low S100A11 expression promoted reactive oxygen species- (ROS-) dependent cell apoptosis through the activation of phospholipase A2 (PLA2), thereby enhancing the sensitivity of lung cancer cells to cisplatin, 5-fluorouracil, and oxaliplatin. In this study, all patients except the one with FIGO stage IG1 cancer received paclitaxel plus platinum-based adjuvant chemotherapy after cytoreductive surgery. The results showed that elevated levels of S100A11 and MMP-9 were associated with chemoresistance. This finding is consistent with previous experimental results, suggesting that S100A11 and MMP9 are potential therapeutic targets for drug-resistant ovarian cancer. Therefore, the joint analysis of S100A11 and MMP-9 is very important in the study of drug-resistance mechanisms in ovarian cancer.

\section{Conclusions}

The results of this study showed that S100A11 and MMP-9 expression in the peripheral blood of EOC patients was significantly higher than that in patients with benign ovarian cancer and healthy controls. S100A11 and MMP-9 levels were low in the control group but were elevated in earlystage tumors and reached the highest level in advanced tumors. Statistical analysis showed that high expression of S100A11 and MMP-9 was positively correlated with the FIGO stage and lymph node metastasis of EOCs, suggesting that S100A11 and MMP-9 play important roles in the occurrence and development of EOCs and are closely associated with prognosis, thus suggesting a new therapeutic target for ovarian cancer. However, whether S100A11 and MMP-9 can be used as a biological indicator for early screening and the long-term postoperative follow-up of malignant changes in EOC still deserves further exploration. Our next step is to investigate the biological roles and mechanisms of S100A11 and MMP-9 in EOCs to assist in clinical diagnosis and treatment and to improve the prognosis of patients.

\section{Data Availability}

All the data can be available by asking from the corresponding author.

\section{Conflicts of Interest}

The authors declare that they have no competing interests.

\section{Acknowledgments}

This research was supported by the Projects of medical and health technology development program in Shandong province (2016WS0279).

\section{References}

[1] L. Mirandola, J. M. Cannon, E. Cobos et al., "Cancer testis antigens: novel biomarkers and targetable proteins for ovarian cancer," International Reviews of Immunology, vol. 30, no. 23, pp. 127-137, 2011.

[2] R. Ignacio and R. C. Bast, "Minireview: human ovarian cancer: biology, current management, and paths to personalizing therapy," Endocrinology, vol. 153, pp. 1593-1602, 2012.

[3] S. A. Koh and K. H. Lee, "HGF-mediated S100A11 overexpression enhances proliferation and invasion of gastric cancer," American Journal of Translational Research, vol. 10, pp. 3385-3394, 2018.

[4] K. Kessenbrock, V. Plaks, and Z. Werb, "Matrix metalloproteinases: regulators of the tumor microenvironment," Cell, vol. 141, no. 1, pp. 52-67, 2010.

[5] Y. Li and J. Zhang, "Expression of S100A11 is a prognostic factor for disease-free survival and overall survival in patients with high-grade serous ovarian cancer," Applied Immunohistochemistry \& Molecular Morphology, vol. 25, no. 2, pp. 110-116, 2015.

[6] J. S. Berek, S. T. Kehoe, L. Kumar, and M. Friedlander, "Cancer of the ovary, fallopian tube, and peritoneum," International Journal of Gynaecology and Obstetrics, vol. 143, pp. 59-78, 2018.

[7] A. M. Chudecka-Głaz, A. A. Cymbaluk-Płoska, J. L. Menkiszak, A. Sompolska-Rzechuła, A. Tołoczko-Grabarek, and I. Rzepka-Górska, "Serum HE4, CA125, YKL-40, bcl-2, cathepsin-L and prediction optimal debulking surgery, response to chemotherapy in ovarian cancer," Journal of ovarian research, vol. 7, no. 1, p. 62, 2014.

[8] N. N. Nik, R. Vang, I. M. Shih, and R. J. Kurman, "Origin and pathogenesis of pelvic (ovarian, tubal, and primary peritoneal) serous carcinoma," Annual Review of Pathology Mechanisms of Disease, vol. 9, no. 1, pp. 27-45, 2014.

[9] T. Adhikary, A. Wortmann, F. Finkernagel et al., "Interferon signaling in ascites-associated macrophages is linked to a favorable clinical outcome in a subgroup of ovarian carcinoma patients," BMC Genomics, vol. 18, no. 1, p. 243, 2017.

[10] M. B. Daly, R. Pilarski, M. B. Yurgelun et al., "NCCN guidelines insights: genetic/familial high-risk assessment: 
breast, ovarian, and pancreatic, version 1.2020," Journal of the National Comprehensive Cancer Network, vol. 18, no. 4, pp. 380-391, 2020.

[11] B. O. Schnekess and M. P. Walsh, "Molecular cloning and expression of avian smooth muscle S100A11 (calgizzarin, S100C)," Biochemistry and Cell Biology, vol. 75, no. 6, pp. 771-775, 1997.

[12] M. C. Anania, C. Miranda, M. G. Vizioli et al., "S100A11 overexpression contributes to the malignant phenotype of papillary thyroid carcinoma," The Journal of Clinical Endocrinology and Metabolism, vol. 98, no. 10, pp. E1591-E1600, 2013.

[13] S. Meding, B. Balluff, M. Elsner et al., "Tissue-based proteomics reveals FXYD3, S100A11 and GSTM3 as novel markers for regional lymph node metastasis in colon cancer," The Journal of Pathology, vol. 228, no. 4, pp. 459-470, 2012.

[14] M. B. Xiao, F. Jiang, W. K. Ni et al., "High expression of S100A11 in pancreatic adenocarcinoma is an unfavorable prognostic marker," Medical Oncology, vol. 29, no. 3, pp. 1886-1891, 2012.

[15] X. G. Liu, X. P. Wang, W. F. Li et al., "Ca2+-binding protein S100A11: a novel diagnostic marker for breast carcinoma," Oncology Reports, vol. 23, no. 5, pp. 1301-1308, 2010.

[16] J. Shankar, A. Messenberg, J. Chan, T. M. Underhill, L. J. Foster, and I. R. Nabi, "Pseudopodial actin dynamics control epithelial-mesenchymal transition in metastatic cancer cells," Cancer Research, vol. 70, no. 9, pp. 3780-3790, 2010.

[17] Y. Liu, X. Han, and B. Gao, "Knockdown of S100A11 expression suppresses ovarian cancer cell growth and invasion," Experimental and Therapeutic Medicine, vol. 9, no. 4, pp. 1460-1464, 2015.

[18] H. Huang, "Matrix metalloproteinase-9 (MMP-9) as a cancer biomarker and MMP-9 biosensors: recent advances," Sensors, vol. 18, no. 10, p. 3249, 2018.

[19] M. Wood, K. Fudge, J. L. Mohler et al., "In situhybridization studies of metallo Proteineses 2 and 9 and TIMP-1 and TIMP-2 expression in human prostate cancer," Clinical \& experimental metastasis, vol. 15, no. 3, pp. 246-258, 1997.

[20] R. G. Moore and S. Maclaughlan, "Current clinical use of biomarkers for epithelial ovarian cancer," Current Opinion in Oncology, vol. 22, no. 5, pp. 492-497, 2010.

[21] H. Huang, Y. J. Li, C. Y. Lan et al., "Clinical significance of ascites in epithelial ovarian cancer," Neoplasma, vol. 60, no. 5, pp. 546-552, 2013.

[22] M. Yin, C. Li, X. Li et al., "Over-expression of LAPTM4B is associated with poor prognosis and chemotherapy resistance in stages III and IV epithelial ovarian cancer," Journal of Surgical Oncology, vol. 104, no. 1, pp. 29-36, 2011.

[23] A. Laios, B. M. Mohamed, L. Kelly et al., "Pre-treatment of platinum resistant ovarian cancer cells with an MMP9/MMP-2 inhibitor prior to cisplatin enhances cytotoxicity as determined by high content screening," International Journal of Molecular Sciences, vol. 14, no. 1, pp. 2085-2103, 2013.

[24] A. Zagryazhskaya, O. Surova, N. S. Akbar et al., "Tudor staphylococcal nuclease drives chemoresistance of non-small cell lung carcinoma cells by regulating S100A11," Oncotarget, vol. 6, no. 14, pp. 12156-12173, 2015. 